\title{
Genetic Diversity Analysis among Indian Mustard (Brassica juncea L. Czern \& Coss) Genotypes under Rainfed Condition
}

\author{
Khushboo Chandra*, Anil Pandey and S.B. Mishra \\ Department of Plant Breeding and Genetics, Dr. Rajendra Prasad Central Agricultural \\ University, Pusa (Samatipur), Bihar - 848125, India \\ *Corresponding author
}

\section{Keywords}

Brassica juncea L. Genetic divergence, $\mathrm{D}^{2}$ analysis, Tocher and Euclidean analysis

\section{Article Info}

Accepted: 04 February 2018 Available Online: 10 March 2018

\section{A B S T R A C T}

An experiment on Indian mustard (Brassica juncea L. Czern and Coss), for divergence studies under rainfed condition, was conducted in Randomized Complete Block Design (RBCD) accommodating 50 genotypes, from various Rapeseed and Mustard centres located across country, randomly in three replications during Rabi 2015-16 at the research farm of Tirhut College of Agriculture, Dholi, Muzaffarpur. Analysis of variance revealed considerably exploitable variability for all the 25 traits. Euclidean and Tocher clustering methods, accommodated Rajendra Suphlam in oligo-genotypic cluster VIII as most divergent genotype. Rearranging fence - sitter genotypes into sub-clusters and rescaled main group through Euclidean method, Varuna and Pusa Bold grouped with Pusa Mahak and exhibited maximum intracluster distance. Utilizing maximum inter-cluster distance between cluster V and VIII followed by IV and VIII and II and VIII altogether 19 crosses suggested. Overall most promising crosses, based on per se and cluster mean values namely RH-0116/ Rajendra Suphlam, PM-25/ Rajendra Suphlam and Kanti/ Rajendra Suphlam were Late $\times$ Early (Days to first flower open, days to $50 \%$ flowering and days to physiological maturity), Non-basal branching $\times$ Basal branching, High $\times$ Low placed siliqua and Low $\times$ High (Harvest - index and Dry matter efficiency) parents along with superiority in several other yield components. Such crosses can provide useful heterotic combinations and could be utilized in trangressive breeding program. Root length followed by height of first primary branch and root volume contributed maximum $(85.39 \%)$ towards total divergence. Geographically unique genotype Rajendra Suphlam proven its overall superiority exhibiting basal branching, deepest tap root with more volume, least height of first siliqua, high yield per plant along with appropriate harvest - index and dry matter efficiency, thus its usefulness in Rainfed agro-ecologies of Bihar.

\section{Introduction}

Oil seed Brassicas, with predominantly cultivated Indian mustard (B. juncea L. Czern and Coss) have major share in edible oil economy of Bihar, offering potential option for diversifying the predominant Rice-Wheat system (Khachatourians et al., 2004) and grown mainly as rainfed / irrigated situations under early, timely and late sown agroecologies. The extent of variability and diversity available decides the success of crop improvement programme making essential to know the spectrum of diversity in any crop 
species and parents based on genetic divergence (Ashana and Pandey, 1980; Ananda and Rawat, 1984). Genetic variability in respect to genetic diversity is the prerequisite for the crop improvement through selection of high yielding progenies. The quantification of genetic diversity by biometrical approaches can help choose diverse parents for a successful hybridization programme, as hybrids between lines of diverse origin generally display a greater heterosis than those between closely related strains (Singh, 1986) and also provides opportunity to obtain the desirable recombinations in the segregating generations (Uddin and Chowdhury, 1994) and could be utilized in transgressive breeding. Evaluation of genetic diversity is important to know the source of genes for a particular trait within the available germplasm (Tomooka, 1991).

Therefore, the present investigation was carried out to determine the divergence among 50 different genotypes of rapeseed mustard.

\section{Materials and Methods}

The experiment consisting of 50 Indian mustard genotypes, including four checks namely, Pusa Mahak (Zonal Check), Varuna (National Check), Pusa Bold (National Check) and Rajendra Suphlam (Local Check) for divergence study, received from different All India Co-ordinated Research ProjectRapeseed and Mustard centres: DRMR, Bharatpur, Rajasthan, CCSHAU, Hisar, Haryana, BARC, Trombay, Maharastra, GBPUAT, Pantnagar, Uttarkhand, CSAUAT, Kanpur, U. P, IARI, NewDelhi, ARS, RAU, Sriganganagar, Rajasthan, DR. RPCAU, Dholi, Bihar, NDUAT, Faizabad, U. P and BAU, Kanke, Ranchi, Jharkhand, was laid out in Randomized Complete Block Design (RCBD) with three replications during Rabi season (2015-16) and was planted on $10^{\text {th }}$ October 2015 under rainfed condition providing only basal dose of fertilizers i. e. $\mathrm{N}$ : $\mathrm{P}_{2} \mathrm{O}_{5}: \mathrm{K}_{2} \mathrm{O}: \mathrm{S}::$ 40: 40: 40: $40 \mathrm{~kg} / \mathrm{ha}$ under residual moisture conditions after the harvest of preceding medium early (110-115 days) paddy cv., Rajendra Bhagwati. At the research farm of Tirhut College of Agriculture, Dholi, Muzaffarpur (Dr. Rajendra Prasad Central Agricultural University, Pusa), Bihar (25. $5^{0}$ $\mathrm{N}, 85.4^{0} \mathrm{E}$ and $52.12 \mathrm{~m} \mathrm{MSL}$ ) in Loam soil (8. $4 \mathrm{pH})$. Each plot was consisted four rows of $5.0 \mathrm{~m}$ length keeping row to row and plant to plant distance $30 \mathrm{~cm}$ and $10 \mathrm{~cm}$, respectively. The spacing between plants was maintained at $10 \mathrm{~cm}$ by thinning at 14 DAS.

Meteorological data (Kharif and Rabi 201516) reflected that the experiment was sown, on residual moisture condition, as the preceding Kharif crop rice has received 697. $20 \mathrm{~mm}$ rainfall distributed in 25 rainy days between June to September $\left(23^{\text {rd }}\right.$ to $38^{\text {th }}$ meteorological weeks 2015). After that experiment at all its phenological stages of Indian mustard crop has not received any rainfall.

The observations were recorded for days to first flower open, days to $50 \%$ flowering, days to physiological maturity, primary branches plant $^{-1}$, secondary branches plant ${ }^{-1}$, number of siliqua plant ${ }^{-1}$, length of siliqua, stem girth, internode length, height of the plant, number of siliqua on primary mother axis, height of first primary branch, height of first siliqua, angle of branch, angle of siliqua, number of seeds siliqua ${ }^{-1}$, root volume, root length, root girth, 1000 seed weight, biological yield, harvest index, oil content and dry matter efficiency and grain yield /plant. The data were recorded on five randomly selected plants from each genotype in each replication leaving the border rows to avoid the sampling error. The observations were recorded using standard methodology. Readings from five plants were averaged replication-wise and the mean data was subjected statistical analyse for yield and its attributing traits. 
Amongst various classificatory analyses, utilized to understand workable variability, $\mathrm{D}^{2}$ - statistic (Mahalonobis, 1928, 1935) using Tocher method (Tocher Rao, 1952) and Euclidean method (Rao, 1952) based on wards' minimum variance dendogram are successfully utilized by various crop breeders for clustering and quantitative measurement of divergence among the genotypes (varieties, strains, mutants, ECs, ICs, etc.) Mahalanobis (1936) $\mathrm{D}^{2}$ - statistic was used for assessing genetic divergence among the test entries. The clustering of $\mathrm{D}^{2}$ values was formed by using generalized distance based- Tocher's method as described by Rao (1952) and also by using Non- hierarchical Euclidean cluster analysis (Beale, 1969; Katyal et al., 1985) was conducted using computer package (Windostat version 8. 5) whereas the formula given by Singh and Choudhary (1977) was utilized for the calculation of intra and inter - cluster distances.

\section{Results and Discussion}

The analysis of variance revealed highly significant differences among the genotypes for all the 25 traits under study, reflecrting presence of considerable variability and genetic worth of the genotypes. Thus, providing adequate scope for selection of superior genotypes aimed at utilizing exploitable variability for enhancing genetic yield potential under rainfed condition of Brassica juncea.

50 Indian mustard genotypes, based on tocher method were grouped in eight different clusters (Table 1). Highest number of genotypes (24) were accommodated in cluster I followed by cluster III (11), cluster II (8), cluster III (3) whereas clusters IV, VI, VII and VIII were oligo-genotypic. Such grouping of genotypes into clusters by Tochers method are based on generalized distance which is statistic related to the coefficient of racial likeliness developed by Mahalanobis (1936) and Rao (1952). More precise clustering method is non-hierarchical Euclidean method (based on Wards minimum variance dendogram) which more critically identifies sub clusters of the main groups at different levels, thus offering additional opportunity to crop breeders, in more critically planning the hybridization programme, using diverse parents aimed at genetic enhancement of any crop species, including crop Brassicas. Euclidean method also accommodated these genotypes in eight different clusters (Table 2) but the genotypes in the clusters, instead of generalized distance used in Tocher method the relative association among the different genotypes is presented in the form of wards minimum variance dendogram, which was prepared using rescaled distance in Euclidean method.

Brassica scientists have utilized these approaches based on generalized distance (Tocher method) and more precisely on rescaled distance (Euclidean method) for selecting diverse potential lines and subsequent utilization, there off, in hybridization - selection breeding program. Highest number of genotypes were in cluster II (14) followed by cluster III (9), cluster V (8), cluster I (6), cluster VI (5), cluster IV (4), cluster VII (3) and cluster VIII which was oligo-genotypic. From both the methods of clustering only rewardive genotype in oligogenotypic cluster was Rajendra Suphlam (VIII in both Euclidean and Tocher method). Among 50 studied, the only dissimilar genotype, namely Rajendra Suphlam have exhibited diversity might be due to geographical uniqueness of this genotype than others.

It is very clear from the perusal of clustering pattern of 50 genotypes by Euclidean and Tocher method that three genotypes, namely Varuna (in mono-genotypic cluster VI) and 
Pusa Bold (V) by Tocher method (fence-sitter genotypes) accommodated in one cluster (VII) in Euclidean method with Pusa Mahak forming sub cluster (among Pusa Bold and Varuna) with main group of three genotypes. This is also noteworthy that cluster VII (Euclidean method) exhibited maximum intracluster distance. Similarly fence-sitter genotypes RGN-13, Divya, TM-2 (III by Tocher method) accommodated with oligogenotype (RH-0116 by Tocher method) in cluster IV (all four genotypes) by Euclidean method. Largest cluster I with 24 genotypes in Tocher method whereas 14 genotypes in cluster II of Euclidean method. Cluster I of Tocher method rescaled and placed 6, 14 and 4 fence-sitter genotypes wholly in Euclidean cluster I and II and partly in III cluster, respectively. The two sub-clusters exhibited similarity between RH-0406 and RGN-13; and between Divya andTM-2 thus more precisely explaining the diversity of the genotypes studied using Euclidean method which could be utilized for diverse parents selection process.

Maximum inter-cluster distance between cluster V and VIII (5970. 024) followed by IV and VIII (5742. 101) and II and VIII (4549. 622) from Euclidean method exhibiting 7, 3 and 14, altogether 24 crosses (Table 3) respectively. Whereas, between cluster III and VIII (1985. 184) followed by IV and VIII (1739. 174), I and VIII (1411. 921), III and VIII (1182. 809) and IV and VIII (1079. 075) with $6,1,5,6$ and 1, altogether 19 divergent crosses (Table 4) respectively from Tocher method reflected that crosses involving genotypes from these cluster will be beneficial from Tocher method, in general whereas Euclidean method, in particular. Thus, hybridization programme, shall be formulated in such a way that the parents belonging to clusters with maximum divergence could be utilized in heterosis breeding and could throw transgressive segregants in $\mathrm{F}_{2}$ generation.
Such genotypes may be chosen from widely separated clusters (Fig. 1 and 2), for crossing programme to get benefits in desirable directions as per breeding objectives. There was no parallelism between genetic diversity and their geographic distributions as the genotypes from one or other geographical regions were grouped together in same cluster and developed from same organization were placed in different clusters might be due to free exchange of genetic materials between clusters and regions and also the number of studied traits and parentage/methodology (For Example induced mutagenesis) involved highly influenced group constellation of 50 genotypes Similar results were observed by Khan (2000), Kumar et al., (2000 a) and Kumar et al., (2000 b).

On comparing generalized distance based Tocher method and precise rescaled subcluster forming Euclidean method (Table 6) 19 promising divergent crosses suggested. Among these crosses as one of the parent the only common oligo-genotypic cluster VIII with Rajendra Suflam proved its uniqueness whereas Pusa Mahak (oligognotypic in cluster VII Tocher method) and cluster VII (along with two other genotypes Varuna and PusaBold in Euclidean method) were most divergent and the crosses based on inter cluster distance involving these genotypes could give heterotic combination for enhancement of yield; and in $\mathrm{F}_{2}$ generations could throw usefully desirable transgressive segregants for rainfed Indian mustard genetic enhancement. Three diverse genotypes, RH0406, Pusa Mahak and Rajendra Suflam superior based on both method can be utilized as testers and crossed with 7 divergent genotypes as lines (Divya, TM-2, RH0116, PM-25, Kanti, Rohini and RGN-13) based on both Tocher and Euclidean method which can be further utilized in hybridization selection breeding programme to get most useful segregants. 
Table.1 Clustering pattern of 50 genotypes of Indian mustard genotypes on the basis of Tocher method

\begin{tabular}{|c|c|c|c|}
\hline $\begin{array}{l}\text { Cluster } \\
\text { No. }\end{array}$ & $\begin{array}{l}\text { Intra cluster } \\
\text { distance }\end{array}$ & $\begin{array}{l}\text { No. of Genotypes } \\
\text { within cluster }\end{array}$ & Genotypes in cluster \\
\hline I & 21.469 & 24 & $\begin{array}{l}\text { NDRE-7, PKRS-28, PM-28 (NPJ-124), KMR10-2, PusaTarak (EJ9913), } \\
\text { TM-215, RAURD-212, PM-27, RH-8812, RAURD (E) -1001, PantRai, Pusa } \\
\text { Bahar, TPM-1, RH-30, Kranti, PusaAgrani (SEJ-2), NDRE-4, NRC-DR-2, } \\
\text { Krishna, TM-4, Basanti, Shivani, RAURD-78, BAUM08-57, }\end{array}$ \\
\hline II & 25.408 & 8 & $\begin{array}{l}\text { DRMRLEJ902, RH-8814, TPM-128, Maya, TM-151, KMR10-1, BAUM08- } \\
\text { 56, DRMR150-35 }\end{array}$ \\
\hline III & 37. 321 & 11 & $\begin{array}{l}\text { RGN-13, Divya, TM-2, RH-0116, PM-25, Kanti, RohinI, RAURD (E) - } \\
\text { 1002, RH-0701, RAURD-214, RGN-48 }\end{array}$ \\
\hline IV & 0.000 & 1 & RH-0406 \\
\hline $\mathbf{V}$ & 32. 277 & 3 & RH-8701, RH-0819, Pusa Bold \\
\hline VI & 0.000 & 1 & Varuna \\
\hline VII & 0.000 & 1 & Pusa Mahak (JD-6) \\
\hline VIII & 0.000 & 1 & Rajendra Suphlam \\
\hline
\end{tabular}

Table.2 Clustering pattern of 50 genotypes of Indian mustard genotypes on the basis of non hierarchical Euclidean method

\begin{tabular}{|c|c|c|c|}
\hline $\begin{array}{l}\text { Cluster } \\
\text { No. }\end{array}$ & $\begin{array}{l}\text { Intra cluster } \\
\text { distance }\end{array}$ & $\begin{array}{l}\text { No. of Genotypes } \\
\text { within cluster }\end{array}$ & Genotypes in cluster \\
\hline $\mathbf{I}$ & 31.609 & 6 & $\begin{array}{l}\text { NDRE-7, PKRS-28, PM-28 (NPJ-124), KM R10-2, Pusa Tarak (EJ9913), } \\
\text { TM215 }\end{array}$ \\
\hline II & 26. 671 & 14 & $\begin{array}{l}\text { RAURD-212, PM-27, RH-8812, RAURD (E) -1001, Pant Rai, Pusa } \\
\text { Bahar, TPM-1, RH-30, Kranti, Pusa Agrani (SEJ-2), NDRE-4, NRC-DR- } \\
\text { 2, Krishna, TM-4 }\end{array}$ \\
\hline III & 48.775 & 9 & $\begin{array}{l}\text { DRMRLEJ902, RH-8814, KMR10-1, BAUM08-56, TPM-128, Basanti, } \\
\text { Shivani, RAURD-78, BAUM08-57 }\end{array}$ \\
\hline IV & 56. 081 & 4 & RH-0406, RGN-13, DIVYA, TM-2 \\
\hline$\overline{\mathbf{V}}$ & 53.913 & 8 & $\begin{array}{l}\text { RH-0116, PM25, Kanti, Rohini, RAURD (E) -1002, RH-0701, RAURD- } \\
\text { 214, RGN-48 }\end{array}$ \\
\hline VI & 80.649 & 5 & TM-151, Maya, DRMR150-35, RH-8701, RH-0819 \\
\hline VII & 219. 294 & 3 & Varuna, Pusa Bold, Pusa Mahak (JD-6) \\
\hline VIII & 0.000 & 1 & Rajendra Suphlam \\
\hline
\end{tabular}

Table.3 Suitable divergent genotypes based on inter cluster distances in Tochers method

\begin{tabular}{|c|c|c|c|c|}
\hline SNO. & $\begin{array}{l}\text { INTER CLUTER } \\
\text { DISTANCE }\end{array}$ & CLUSTERS & DIVERGENT GENOTYPES & $\begin{array}{l}\text { NUMBBER OF } \\
\text { CROSSES }\end{array}$ \\
\hline \multirow[t]{2}{*}{1} & \multirow{2}{*}{ 1985. 184} & III & Divya, TM-2, RH-0116, PM-25, Kanti, Rohini & \multirow[t]{2}{*}{6} \\
\hline & & VIII (O) & Rajendra Suphlam & \\
\hline \multirow[t]{2}{*}{2} & \multirow[t]{2}{*}{ 1739. 174} & IV $(\mathrm{O})$ & RH-0406 & \multirow[t]{2}{*}{1} \\
\hline & & VIII (O) & Rajendra Suphlam & \\
\hline \multirow[t]{2}{*}{3} & \multirow[t]{2}{*}{1411.921} & I & RAURD-212, PM-27, RH-8812, RH-30, Kranti & \multirow[t]{2}{*}{5} \\
\hline & & VIII (O) & Rajendra Suphlam & \\
\hline \multirow[t]{2}{*}{4} & \multirow[t]{2}{*}{ 1182. 809} & III & Divya, TM-2, RH-0116, PM-25, Kanti, Rohini & \multirow[t]{2}{*}{6} \\
\hline & & VII $(\mathrm{O})$ & Pusa Mahak & \\
\hline \multirow[t]{2}{*}{5} & \multirow[t]{2}{*}{ 1079. 075} & IV $(\mathrm{O})$ & RH-0406 & \multirow[t]{2}{*}{1} \\
\hline & & VII $(\mathrm{O})$ & Pusa Mahak & \\
\hline \multicolumn{4}{|c|}{ TOTAL } & 19 \\
\hline
\end{tabular}


Table.4 Suitable divergent genotypes based on inter cluster distances in Euclidean method

\begin{tabular}{|c|c|c|c|c|}
\hline SNO. & $\begin{array}{l}\text { INTER CLUTER } \\
\text { DISTANCE }\end{array}$ & CLUSTERS & DIVERGENT GENOTYPES & $\begin{array}{l}\text { NUMBER OF } \\
\text { CROSSES }\end{array}$ \\
\hline \multirow[t]{2}{*}{1} & \multirow[t]{2}{*}{5970.024} & $\mathrm{~V}$ & RH-0116, PM-25, RGN-48, RH-0701, RAURD (E) -1002, Kanti, Rohini & \multirow[t]{2}{*}{7} \\
\hline & & VIII (O) & Rajendra Suphlam & \\
\hline \multirow[t]{2}{*}{2} & \multirow[t]{2}{*}{ 5742. 101} & IV & RH0406, RGN-13, Divya, TM-2 & \multirow[t]{2}{*}{4} \\
\hline & & VIII (O) & Rajendra Suphlam & \\
\hline \multirow[t]{2}{*}{3} & \multirow[t]{2}{*}{4549.622} & II & $\begin{array}{l}\text { RAURD-212, PM-27, RH-8812, RAURD (E) -1001, Pant Rai, Pusa Bahar, TPM-1, RH-30, } \\
\text { Kranti, Pusa Agrani (SEJ-2), NDRE-4, NRC-DR-2, Krishna, TM-4 }\end{array}$ & \multirow[t]{2}{*}{14} \\
\hline & & VIII (O) & Rajendra Suphlam & \\
\hline \multirow[t]{2}{*}{4} & \multirow[t]{2}{*}{3981.891} & I & NDRE-7, PKRS-28, PM-28 (NPJ-124), KM R10-2, Pusa Tarak (EJ9913), TM-215 & \multirow[t]{2}{*}{6} \\
\hline & & VIII $(\mathrm{O})$ & Rajendra Suphlam & \\
\hline \multirow[t]{2}{*}{5} & \multirow[t]{2}{*}{3270.975} & III & $\begin{array}{c}\text { DRMRLEJ902, RH-8814, KMR10-1, BAUM08-56, TPM-128, Basanti, ShivanI, RAURD-78, } \\
\text { BAUM08-57 }\end{array}$ & \multirow[t]{2}{*}{9} \\
\hline & & VIII $(\mathrm{O})$ & Rajendra Suphlam & \\
\hline \multirow[t]{2}{*}{6} & \multirow[t]{2}{*}{2768.430} & IV & RH-0406, RGN-13, Divya, TM-2 & \multirow[t]{2}{*}{9} \\
\hline & & VII & Varuna, Pusa Bold, Pusa Mahak & \\
\hline \multirow[t]{2}{*}{7} & \multirow[t]{2}{*}{ 2764. 117} & $\mathrm{~V}$ & RH-0116, PM-25, RGN-48, RH-0701, RAURD (E) -1002, Kanti, Rohini & \multirow[t]{2}{*}{24} \\
\hline & & VII & Varuna, Pusa Bold, Pusa Mahak & \\
\hline & & & TOTAL & 73 \\
\hline
\end{tabular}

Table.5 Comparisons of Diverse Brassica juncea genotypes based on genetic distance, cluster mean and superior per se performance for earliness, oil content and seed yield component traits. (Tochers and Euclidean method)

\begin{tabular}{|c|c|c|}
\hline \multicolumn{2}{|l|}{ Characters } & Cluster \\
\hline \multirow{2}{*}{$\begin{array}{l}\text { Days to First Flower } \\
\text { Open }\end{array}$} & Early & VIII \\
\hline & Late & III \\
\hline \multirow[t]{2}{*}{ Days to $50 \%$ flowering } & Early & VII, VIII \\
\hline & Late & III \\
\hline \multirow{2}{*}{$\begin{array}{l}\text { Days to physiological } \\
\text { maturity }\end{array}$} & Early & VIII \\
\hline & Late & III \\
\hline Primary branches plant ${ }^{-1}$ & & VII \\
\hline $\begin{array}{l}\text { Secondary branches } \\
\text { plant }^{-1}\end{array}$ & & VII \\
\hline Number of siliqua plant ${ }^{-1}$ & & VII \\
\hline Length of siliqua & & VIII \\
\hline Stem girth & & VIII \\
\hline \multirow[t]{2}{*}{ Internode length } & Low & III \\
\hline & High & VIII \\
\hline \multirow[t]{2}{*}{ Height of the plant } & Tall & VIII \\
\hline & Dwarf & III \\
\hline $\begin{array}{l}\text { Number of siliqua on } \\
\text { primary mother axis }\end{array}$ & & VIII \\
\hline \multirow[t]{2}{*}{$\begin{array}{l}\text { Height of first primary } \\
\text { branch }\end{array}$} & $\begin{array}{l}\text { Non-basal } \\
\text { branching }\end{array}$ & VII \\
\hline & $\begin{array}{l}\text { Basal } \\
\text { branching }\end{array}$ & VIII \\
\hline \multirow[t]{2}{*}{ Height of first siliqua } & High position & III \\
\hline & Lower position & VIII \\
\hline Angle of branch & Compact & VIII \\
\hline Angle of siliqua & Semi- apressed & VIII \\
\hline Number of seeds siliqua $^{-1}$ & & VIII \\
\hline Root volume & & VIII \\
\hline Root length & & VIII \\
\hline Root girth & & VIII \\
\hline 1000 Seed weight & & VIII \\
\hline Biological yield & & VIII \\
\hline Harvest index & & VII \\
\hline Oil content & & VIII \\
\hline Dry matter efficiency & & VII \\
\hline Grain yield /plant & & VIII \\
\hline
\end{tabular}

\begin{tabular}{|c|c|}
\hline Suitable Parents & Cluster \\
\hline Rajendra suphlam & VIII \\
\hline $\begin{array}{l}\text { Divya, Kanti, PM-25, } \\
\text { Rohini, TM-2 }\end{array}$ & V \\
\hline $\begin{array}{l}\text { Pusa Mahak, Rajendra } \\
\text { suphlam }\end{array}$ & VIII \\
\hline $\begin{array}{l}\text { Divya, Kanti, PM-25, } \\
\text { Rohini, TM-2 }\end{array}$ & V \\
\hline Rajendra suphlam & VIII \\
\hline $\begin{array}{l}\text { Divya, Kanti, PM-25, } \\
\text { Rohini, TM-2 }\end{array}$ & V \\
\hline Pusa Mahak & VIII \\
\hline Pusa Mahak & VIII \\
\hline Pusa Mahak & VIII \\
\hline Rajendra suphlam & VIII \\
\hline Rajendra suphlam & VIII \\
\hline $\begin{array}{l}\text { Divya, Kanti, PM-25, } \\
\text { Rohini, TM-2 }\end{array}$ & V \\
\hline Rajendra suphlam & VIII \\
\hline Rajendra suphlam & VIII \\
\hline $\begin{array}{l}\text { Divya, Kanti, PM-25, } \\
\text { Rohini, TM-2 }\end{array}$ & V \\
\hline Rajendra suphlam & VIII \\
\hline Pusa Mahak & V \\
\hline Rajendra suphlam & VIII \\
\hline $\begin{array}{l}\text { Divya, Kanti, PM-25, } \\
\text { Rohini, TM-2 }\end{array}$ & V \\
\hline Rajendra suphlam & VIII \\
\hline Rajendra suphlam & VIII \\
\hline Rajendra suphlam & VIII \\
\hline Rajendra suphlam & VIII \\
\hline Rajendra suphlam & VIII \\
\hline Rajendra suphlam & VIII \\
\hline Rajendra suphlam & VIII \\
\hline Rajendra suphlam & VIII \\
\hline Rajendra suphlam & VIII \\
\hline Pusa Mahak & VII \\
\hline Rajendra suphlam & VIII \\
\hline Pusa Mahak & VII \\
\hline Rajendra suphlam & VIII \\
\hline
\end{tabular}

\begin{tabular}{|l|l|}
\hline Suitable Parents \\
\hline Rajendra suphlam \\
\hline RH-0116, PM-25, Kanti, RH-0701, \\
RGN-48, RAURD (E) -1002 \\
\hline Rajendra suphlam \\
\hline RH-0116, PM-25, Kanti, RH-0701, \\
RGN-48, RAURD (E) -1002 \\
\hline Rajendra suphlam \\
\hline RH-0116, PM-25, Kanti, RH0701, RGN- \\
48, RAURD (E) -1002 \\
\hline Rajendra suphlam \\
\hline Rajendra suphlam \\
\hline Rajendra suphlam \\
\hline Rajendra suphlam \\
\hline Rajendra suphlam \\
\hline RH0116, PM-25, Kanti, RH0701, \\
RGN48, RAURDE-1002 \\
\hline Rajendra suphlam \\
\hline Rajendra suphlam \\
\hline RH-0116, PM-25, Kanti, RH-0701, \\
RGN-48, RAURD (E) -1002 \\
\hline Rajendra suphlam \\
\hline RH-0116, PM-25, Kanti, RH-0701, \\
RGN-48, RAURD (E) -1002 \\
\hline Rajendra suphlam \\
\hline RH-0116, PM-25, Kanti, RH-0701, \\
RGN-48, RAURD (E) -1002 \\
\hline Rajendra suphlam \\
\hline Rajendra suphlam \\
\hline Rajendra suphlam \\
\hline Rajendra suphlam \\
\hline Rajendra suphlam \\
\hline Rajendra suphlam \\
\hline Rajendra suphlam \\
\hline Rajendra suphlam \\
\hline Rajendra suphlam \\
\hline Varuna, Pusa Bold, Pusa Mahak \\
\hline Vajendra suphlam \\
\hline
\end{tabular}

\begin{tabular}{|c|c|}
\hline Common Parents & per se Performance \\
\hline Rajendra suphlam & Rajendra suphlam (35.67*) \\
\hline Kanti, PM-25 & RH-0116 (42. 67) \\
\hline Rajendra suphlam & Rajendra suphlam (95.00**) \\
\hline PM-25, Kanti & $\begin{array}{l}\text { PM-25 (103.00) } \\
\text { Kanti (103.00) }\end{array}$ \\
\hline Rajendra suphlam & Rajendra suphlam $\left(123.33^{* *}\right)$ \\
\hline Kanti, PM-25 & Kanti (133.00) \\
\hline- & Pusa Mahak (13.20**) \\
\hline - & Pusa Mahak (26. 12**) \\
\hline- & Pusa Mahak (1081. 45**) \\
\hline Rajendra suphlam & Rajendra suphlam (6.33**) \\
\hline Rajendra suphlam & Rajendra suphlam $\left(8.40^{* *}\right)$ \\
\hline Kanti, PM-25 & Kanti (7. 73) \\
\hline Rajendra suphlam & Rajendra suphlam (17.08**) \\
\hline Rajendra suphlam & Rajendra suphlam (243. $\left.73^{* *}\right)$ \\
\hline PM-25, Kanti & Kanti (117. 26) \\
\hline Rajendra suphlam & Rajendra suphlam (65.67**) \\
\hline- & Kanti (93.60) \\
\hline Rajendra suphlam & Rajendra suphlam $\left(9.93^{* *}\right)$ \\
\hline PM-25, Kanti & RH-0116 (42. 67) \\
\hline Rajendra suphlam & Rajendra suphlam (35. 67) \\
\hline Rajendra suphlam & Rajendra suphlam (14. 67) \\
\hline Rajendra suphlam & Rajendra suphlam (13.33) \\
\hline Rajendra suphlam & Rajendra suphlam $(12.67 * *)$ \\
\hline Rajendra suphlam & Rajendra suphlam (35.23**) \\
\hline Rajendra suphlam & Rajendra suphlam (24. 56**) \\
\hline Rajendra suphlam & Rajendra suphlam $\left(5.97^{* *}\right)$ \\
\hline Rajendra suphlam & Rajendra suphlam $(7.67 * *)$ \\
\hline Rajendra suphlam & Rajendra suphlam (2416. 67) \\
\hline Pusa Mahak & Pusa Mahak23. 85**) \\
\hline Rajendra suphlam & All are at par \\
\hline Pusa Mahak & Pusa Mahak19. 34**) \\
\hline Rajendra suphlam & Rajendra suphlam (29.33**) \\
\hline
\end{tabular}


Table.6 Suitable common Divergent and less Divergent crosses based on inter cluster distances in Tochers and Euclidean method

\begin{tabular}{|c|c|c|c|c|c|c|}
\hline \multicolumn{7}{|c|}{$\begin{array}{l}\text { PROMISING DIVERGENT CROSSES } \\
\end{array}$} \\
\hline \multicolumn{2}{|r|}{ Tochers method } & \multicolumn{2}{|r|}{ Euclidean method } & \multicolumn{3}{|c|}{$\begin{array}{l}\text { Common promising divergent crosses } \\
\text { Tocher and Euclidean method }\end{array}$} \\
\hline Clusters & Cross & Clusters & Cross & Common Crosses & $\begin{array}{l}\text { Clusters } \\
\text { (Tocher) }\end{array}$ & $\begin{array}{l}\text { Clusters } \\
\text { (Euclidian) }\end{array}$ \\
\hline \multicolumn{2}{|c|}{$\begin{array}{l}\text { Crosses between cluster III (6 genotypes) } \\
\text { and VIII }(0)\end{array}$} & IV×VIII $(\mathrm{O})$ & 1. 1 Divya $\times$ Rajendra suphlam & 1. Divyax Rajendra suphlam & III×VIII (O) & $\begin{array}{l}\text { IV } \times \text { VIII } \\
(\mathrm{O})\end{array}$ \\
\hline $\begin{array}{l}\text { III } \times \text { VIIII } \\
(\mathbf{O})\end{array}$ & $\begin{array}{l}\text { 1. } 1 \text { Divyax Rajendra } \\
\text { suphlam }\end{array}$ & IV×VIII $(\mathrm{O})$ & 1. 2 TM- $2 \times$ Rajendra suphlam & 2. TM-2× Rajendra suphlam & III×VIII (O) & $\begin{array}{l}\text { IV } \times \text { VIII } \\
(\mathrm{O})\end{array}$ \\
\hline $\begin{array}{l}\text { III } \times \text { VIII } \\
(\mathbf{O})\end{array}$ & $\begin{array}{l}\text { 1. } 2 \text { TM-2 } 2 \text { Rajendra } \\
\text { suphlam }\end{array}$ & $\mathrm{IV} \times \mathrm{VIII}(\mathrm{O})$ & 1. 3 RH -0406x Rajendra suphlam & $\begin{array}{l}\text { 3. RH-0116× Rajendra } \\
\text { suphlam }\end{array}$ & III×VIII (O) & $\mathrm{V} \times \mathrm{VIII}(\mathrm{O})$ \\
\hline $\begin{array}{l}\text { III } \times \text { VIII } \\
\text { (O) }\end{array}$ & $\begin{array}{l}\text { 1. } 3 \text { RH-0116× Rajendra } \\
\text { suphlam }\end{array}$ & $\mathrm{IV} \times \mathrm{VIII}(\mathrm{O})$ & 1. 4 RGN-13x Rajendra suphlam & 4. PM-25× Rajendra suphlam & III×VIII (O) & $\mathrm{V} \times \mathrm{VIII}(\mathrm{O})$ \\
\hline $\begin{array}{l}\text { III } \times \text { VIII } \\
(\mathbf{O})\end{array}$ & $\begin{array}{l}\text { 1. } 4 \text { PM-2× Rajendra } \\
\text { suphlam }\end{array}$ & V×VIII (O) & 2. 1 RH-0116× Rajendra suphlam & 5. Kanti× Rajendra suphlam & III×VIII (O) & V×VIII (O) \\
\hline $\begin{array}{l}\text { III } \times \text { VIII } \\
(\mathbf{O})\end{array}$ & $\begin{array}{l}\text { 1. } 5 \text { Kanti× Rajendra } \\
\text { suphlam }\end{array}$ & $\mathrm{V} \times \mathrm{VIII}(\mathrm{O})$ & 2. 2 PM-25× Rajendra suphlam & 6. Rohini× Rajendra suphlam & III×VIII (O) & $\mathrm{V} \times \mathrm{VIII}(\mathrm{O})$ \\
\hline $\begin{array}{l}\text { III } \times \text { VIIII } \\
(\mathbf{O})\end{array}$ & $\begin{array}{l}\text { 1. } 6 \text { Rohini } \times \text { Rajendra } \\
\text { Suflam }\end{array}$ & V×VIII (O) & 2. 3 RGN-48 x Rajendra suphlam & $\begin{array}{l}\text { 7. RH-0406× Rajendra } \\
\text { suphlam }\end{array}$ & $\begin{array}{l}\text { IV }(\mathrm{O}) \times \mathrm{VIII} \\
(\mathrm{O})\end{array}$ & $\begin{array}{l}\text { IV } \times \text { VIII } \\
(\mathrm{O})\end{array}$ \\
\hline \multicolumn{2}{|c|}{$\begin{array}{l}\text { Crosses between cluster IV (O) and VIII } \\
(0)\end{array}$} & V×VIII (O) & 2. 4 RH-0701x Rajendra suphlam & $\begin{array}{l}\text { 8. RAURD-212× Rajendra } \\
\text { suphlam }\end{array}$ & IxVIII (O) & $\mathrm{II} \times \mathrm{VIII}(\mathrm{O})$ \\
\hline $\begin{array}{l}\text { IV (O) } \\
\times \text { VIII (O) }\end{array}$ & $\begin{array}{l}\text { 2. } 1 \text { RH-0406 Rajendra } \\
\text { suphlam }\end{array}$ & $\mathrm{V} \times \mathrm{VIII}(\mathrm{O})$ & $\begin{array}{l}\text { 2. } 5 \text { RAURD (E) - } 1002 \times \text { Rajendra } \\
\text { suphlam }\end{array}$ & 9. PM-27× Rajendra suphlam & $\mathrm{I} \times \mathrm{VIII}(\mathrm{O})$ & II×VIII (O) \\
\hline \multicolumn{2}{|c|}{$\begin{array}{l}\text { Crosses between cluster I (5 genotypes) } \\
\text { and VIII (O) }\end{array}$} & V×VIII (O) & 2. 6 Kanti x Rajendra suphlam & $\begin{array}{l}\text { 10. RH- } 8812 \times \text { Rajendra } \\
\text { suphlam }\end{array}$ & I×VIII (O) & IIxVIII (O) \\
\hline IxVIII (O) & $\begin{array}{l}\text { 3. } 1 \text { RAURD-212x } \\
\text { Rajendra suphlam }\end{array}$ & V×VIII (O) & 2. 7 Rohini x Rajendra suphlam & $\begin{array}{l}\text { 11. RH-30× Rajendra } \\
\text { suphlam }\end{array}$ & $\mathrm{I} \times \mathrm{VIII}(\mathrm{O})$ & $\mathrm{II} \times \mathrm{VIII}(\mathrm{O})$ \\
\hline IxVIII (O) & $\begin{array}{l}\text { 3. } 2 \text { PM- } 27 \times \text { Rajendra } \\
\text { suphlam }\end{array}$ & II×VIII (O) & $\begin{array}{l}\text { 3. } 1 \text { RAURD-212× Rajendra } \\
\text { suphlam }\end{array}$ & 12. Krantix Rajendra suphlam & $\mathrm{I} \times \mathrm{VIII}(\mathrm{O})$ & II×VIII (O) \\
\hline $\mathbf{I} \times$ VIII (O) & $\begin{array}{l}\text { 3. } 3 \text { RH- } 8812 \times \text { Rajendra } \\
\text { suphlam }\end{array}$ & $\mathrm{II} \times \mathrm{VIII}(\mathrm{O})$ & 3. 2 PM-27× Rajendra suphlam & \multicolumn{3}{|c|}{ Sub Total (a) most divergent crosses: 12} \\
\hline $\mathbf{I} \times$ VIII (O) & $\begin{array}{l}\text { 3. } 4 \text { RH-30 Rajendra } \\
\text { suphlam }\end{array}$ & II×VIII (O) & 3. 3 RH- $8812 \times$ Rajendra suphlam & Divyax Pusa & III $\times$ VII $(\mathrm{O})$ & $\mathrm{IV} \times \mathrm{VII}$ \\
\hline $\mathbf{I} \times$ VIII (O) & $\begin{array}{l}\text { 3. } 5 \text { Kranti× Rajendra } \\
\text { suphlam }\end{array}$ & $\mathrm{II} \times \mathrm{VIII}(\mathrm{O})$ & 34. 4 RH-30× Rajendra suphlam & TM-2× Pusa & III $\times$ VII (O) & $\mathrm{IV} \times \mathrm{VII}$ \\
\hline \multicolumn{2}{|c|}{$\begin{array}{l}\text { Crosses between cluster III (6 genotypes) } \\
\text { and VIII }(0)\end{array}$} & $\mathrm{II} \times \mathrm{VIII}(\mathrm{O})$ & 3. 5 Krantix Rajendra suphlam & RH-0116× Pusa & $\mathrm{III} \times \mathrm{VII}(\mathrm{O})$ & $\mathrm{V} \times \mathrm{VII}$ \\
\hline IIIXVII (O) & 4. 1 Divya× Pusa Mahak & $\mathrm{II} \times \mathrm{VIII}(\mathrm{O})$ & $\begin{array}{l}\text { 3. } 6 \text { RAURD (E) 1001x Rajendra } \\
\text { suphlam }\end{array}$ & PM-25× Pusa & $\mathrm{III} \times \mathrm{VII}(\mathrm{O})$ & $\mathrm{V} \times \mathrm{VII}$ \\
\hline III $\times$ VII $(\mathbf{O})$ & 4. 2 TM-2× Pusa Mahak & II×VIII (O) & 3. 7 Pant Rai x Rajendra suphlam & Mahak & III $\times$ VII (O) & $\mathrm{V} \times \mathrm{VII}$ \\
\hline III $\times$ VII $(\mathbf{O})$ & $\begin{array}{l}\text { 4. } 3 \text { RH- } 0116 \times \text { Pusa } \\
\text { Mahak }\end{array}$ & $\mathrm{II} \times \mathrm{VIII}(\mathrm{O})$ & $\begin{array}{l}\text { 3. } 8 \text { Pusa Baharx Rajendra } \\
\text { suphlam }\end{array}$ & Rohini× Pusa & III $\times$ VII (O) & $\mathrm{V} \times \mathrm{VII}$ \\
\hline III $\times$ VII $(0)$ & 4. 4 PM-25× Pusa Mahak & II×VIII (O) & 3. 9 TPM-1 x Rajendra suphlam & RH-0406× Pusa & $\begin{array}{l}\text { IV }(\mathrm{O}) \times \mathrm{VII} \\
(\mathrm{O})\end{array}$ & IV $\times$ VII \\
\hline III $\times$ VII $(\mathbf{O})$ & 4. 5 Kantix Pusa Mahak & $\mathrm{II} \times \mathrm{VIII}(\mathrm{O})$ & $\begin{array}{l}\text { 3. } 10 \text { Pusa Agrani x Rajendra } \\
\text { suphlam }\end{array}$ & & otal (b) dive & crosses: 7 \\
\hline III $\times$ VII (O) & 4. 6 Rohini $\times$ Pusa Mahak & $\mathrm{II} \times \mathrm{VIII}(\mathrm{O})$ & $\begin{array}{l}\text { 3. } 11 \text { NDRE-4 x Rajendra } \\
\text { suphlam }\end{array}$ & & Total diverg & t crosses: 19 \\
\hline $\begin{array}{l}\text { Crosses betv } \\
(\mathbf{O})\end{array}$ & cluster IV (O) and VII & II×VIII (O) & $\begin{array}{l}\text { 3. } 12 \text { NRC-DR-2x Rajendra } \\
\text { suphlam }\end{array}$ & $\begin{array}{l}\text { These crosses are divergent in } \\
\text { Euclidiean method although }\end{array}$ & $\begin{array}{l}\text { Tocher methoc } \\
\text { ey are commo }\end{array}$ & $\begin{array}{l}\text { sut in } \\
\mathrm{n} \mathrm{V} \times \mathrm{VII}\end{array}$ \\
\hline IV $(\mathbf{O}) \times$ VII & $\begin{array}{l}\text { 5. } 1 \text { RH0406x } \\
\text { Pusa Mahak }\end{array}$ & $\mathrm{II} \times \mathrm{VIII}(\mathrm{O})$ & 3. 13 Krishna x Rajendra suphlam & $\begin{array}{l}\text { but less divergent than } \mathrm{I} \times \mathrm{VII} \\
\mathrm{V} \times \mathrm{VIII} \text { in Euclidiean method }\end{array}$ & IxVIII, IV & and \\
\hline & & II×VIII (O) & 3. 14 TM-4x Rajendra suphlam & & & \\
\hline Total & 19 Crosses & Total & 25 Crosses & & & \\
\hline
\end{tabular}


Fig.1 Clustering of 50 Indian mustard genotypes based on Tocher's method

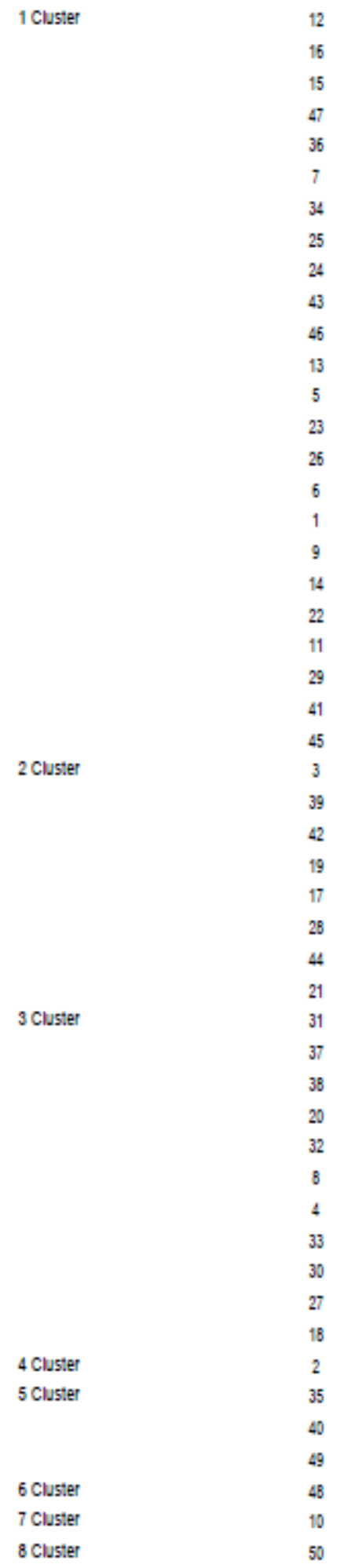

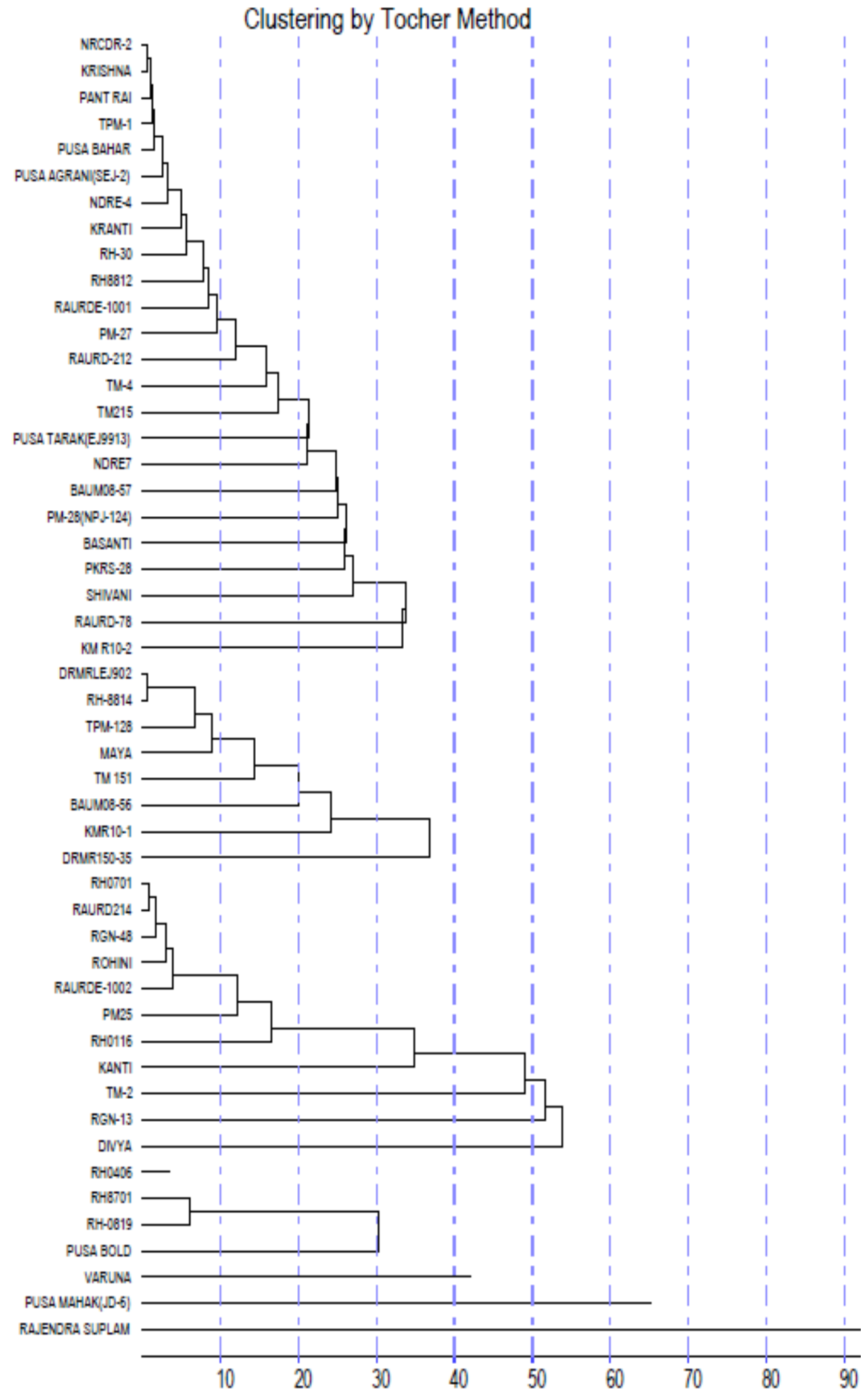


Fig.2 Clustering pattern of 50 Indian mustard genotypes by wards minimum variance dendogram (Euclidean method)
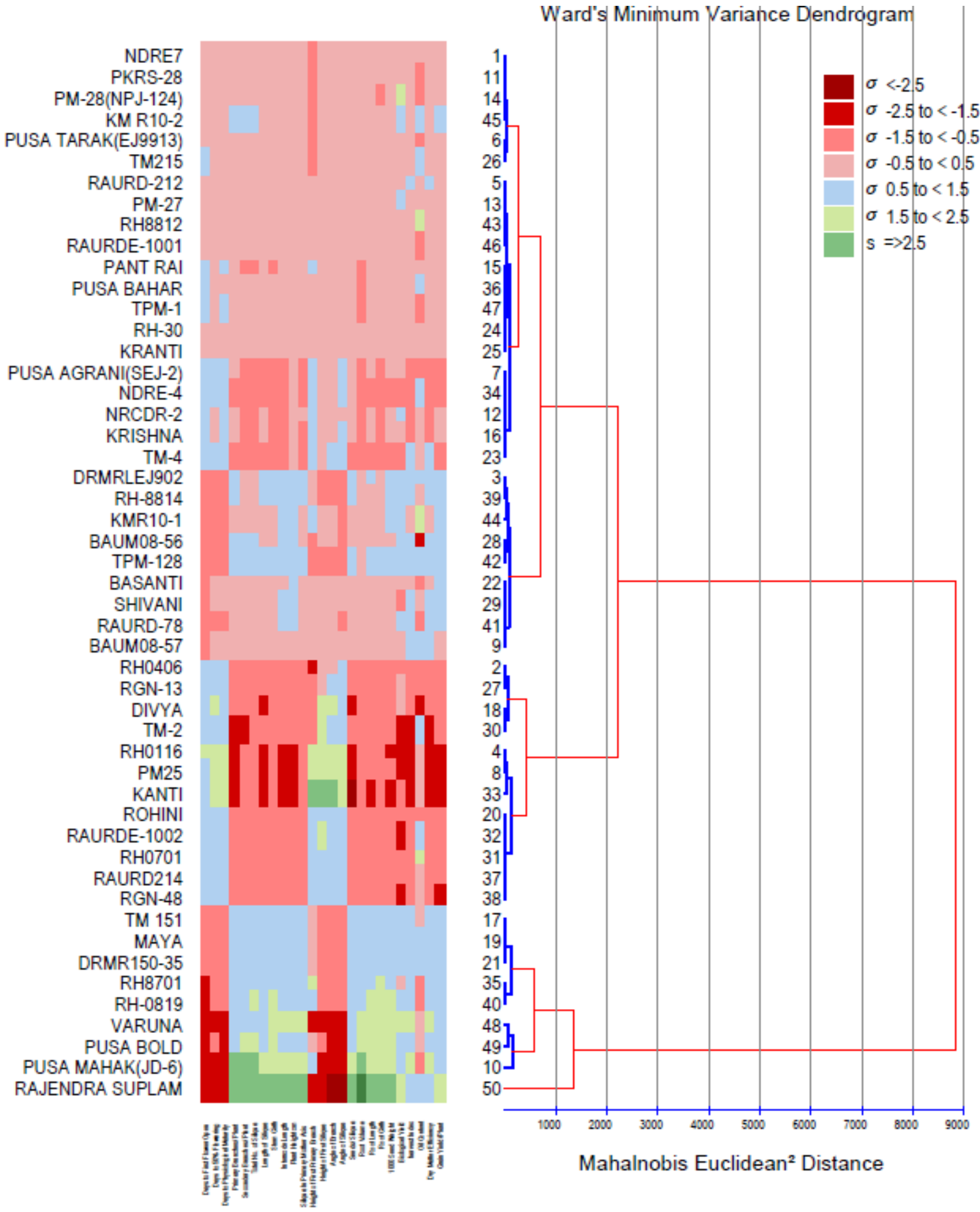
Fig.3 Cluster diagram depicting intra and inter- cluster distances. Fig.4 Cluster diagram depicting intra and inter- cluster distances based on Tocher's method based on Euclidean method
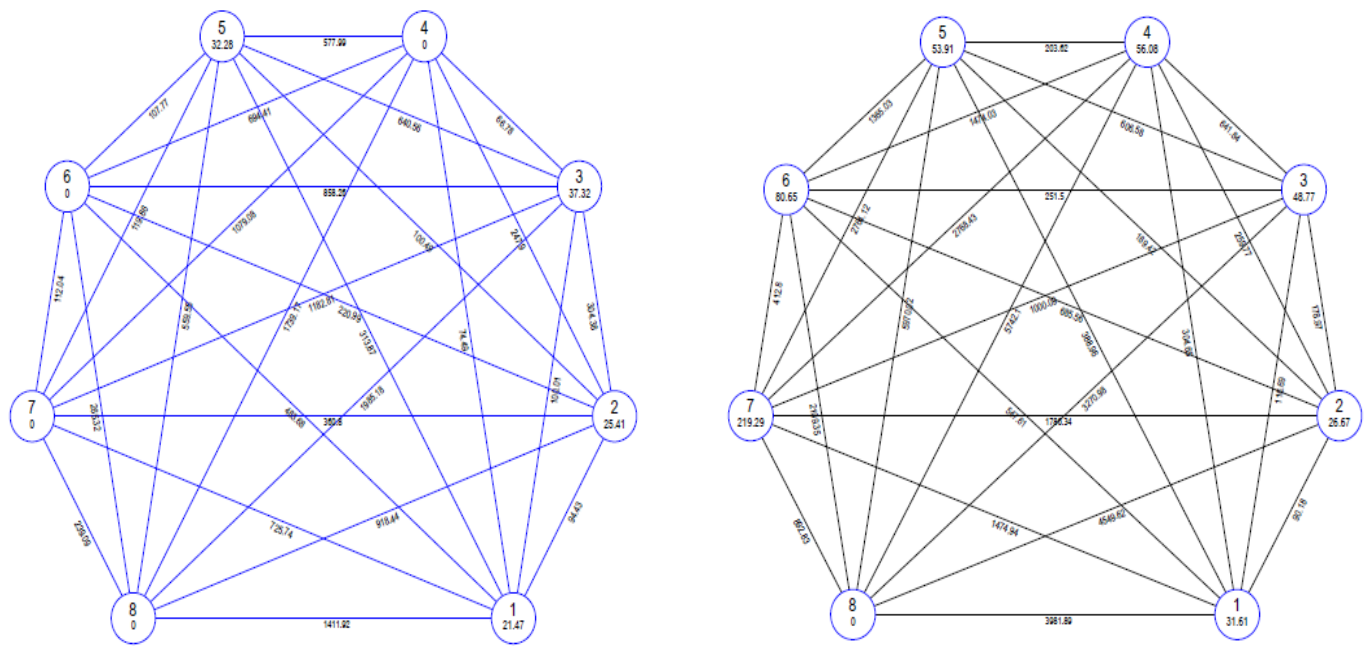

Fig.5 Maximum contribution towards Total divergence

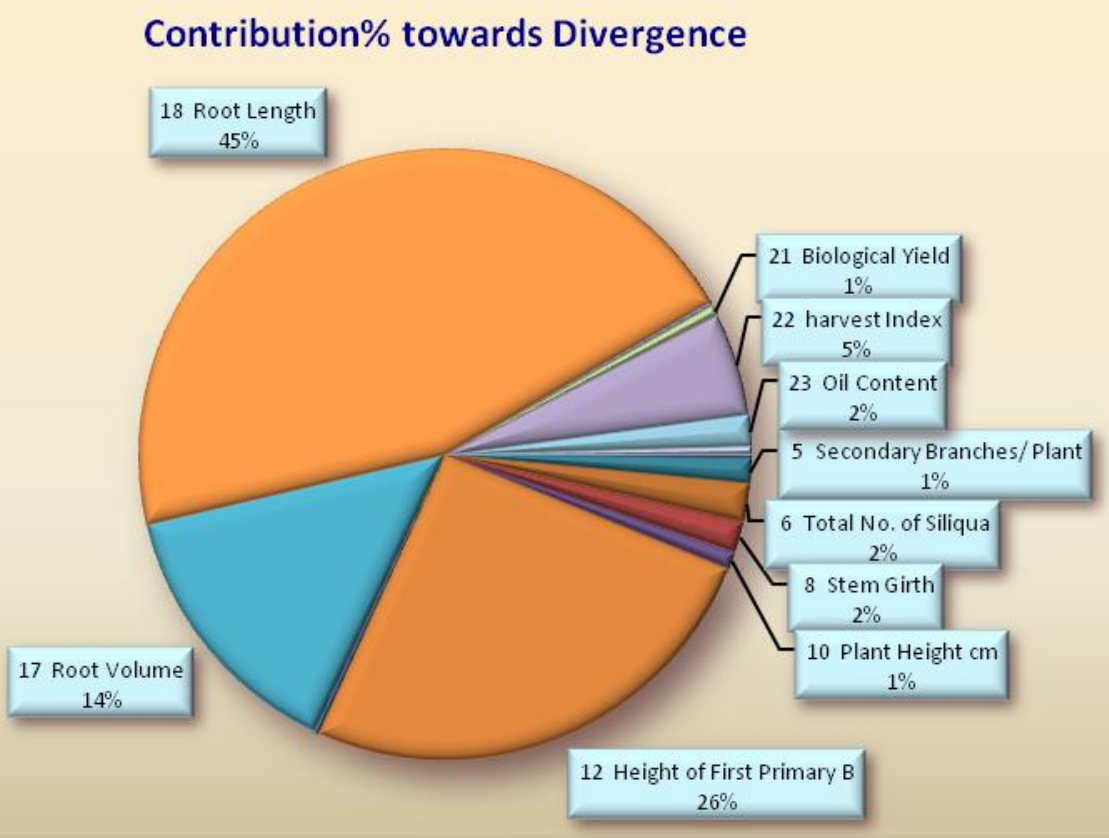

Amongst 19 crosses suggested, 12 crosses involved Divya, TM-2, RH0406 (Euclidean cluster IV) whereas RH-0116, PM-25, Kanti, Rohini, (Euclidean cluster V), RAURD-212, PM-27, RH8812, RH-30 and Kranti (Euclidean cluster II) with Rajendra Suphlam were more divergent common from both Euclidean and Tocher methods. These crosses on the basis of days to first open, days to $50 \%$ flowering and days to physiological maturity further identified as parents involving Late $\times$ Early (RH-0116/Rajendra Suphlam, PM-25/ Rajendra Suphlam, Kanti/ Rajendra Suphlam). On the basis of height of first siliqua (i. e. productive height of the genotype), four crosses involved cross 
between High $\times$ Low position of siliqua (Divya/ Rajendra Suphlam, RH-0116/ Rajendra Suphlam, PM-25/ Rajendra Suphlam and Kanti/ Rajendra Suphlam). Interestingly all 12 except three crosses involved Basal/Non-Basal (based on height of first primary branch $<30 \mathrm{~cm}$ and $>30 \mathrm{~cm}$ as non-basal branching type) whereas three crosses, namely Divya/ Rajendra Suphlam, TM-2/ Rajendra Suphlam and RH-0116/ Rajendra Suphlam involved both basal branching parents in hybridization. Most important but difficult components, namely harvest- index and dry matter efficiency, all these crosses involved Low/High parents as Rajendra Suphlam and Pusa Mahak are only two genotypes with high harvest- index and dry matter efficiency. Out of the studied, 50 genotypes under rainfed (only residual rainfall, no rainfall during different phenological crop growth from seeding to siliqua pre- maturity stage i. e. OctoberFebruary). Overall three crosses namely $\mathrm{RH}-$ 0116/ Rajendra Suphlam, PM-25/ Rajendra Suphlam, Kanti/ Rajendra Suphlam were most promising as they involved Late $\times$ Early (days to first open, days to 50\% flowering and days to physiological maturity), Basal/NonBasal, High $\times$ Low placed siliqua and Low $\times$ High (harvest- index and dry matter efficiency) parents, and could have possibility to exhibit heterosis and could throw transgressive segregants. Additionally, only one cross between Pusa Mahak and Rajendra Suphlam involved, along with superiority in one many other traits, high harvest - index and dry matter efficiency, could be a better option for heterosis breeding for mustard improvement under rainfed condition.

In Tochers method (Table 5) primary branches plant ${ }^{-1}$, secondary branches plant ${ }^{-1}$, number of siliqua plant $^{-1}$, height of first primary branch, harvest index dry matter efficiency highest cluster means fall in cluster VII which is oligo-genotypic accommodating
Pusa Mahak; whereas for length of siliqua, stem girth, internode length, height of the plant, number of siliqua in primary mother axis, angle of branch, angle of siliqua, number of seeds siliqua ${ }^{-1}$, root volume, root length, root girth, 1000 seed weight, biological yield, oil content and grain yield /plant highest cluster means recorded in cluster VIII which is also oligo-genotypic accommodating Rajendra suphlam. On basis of highest cluster mean for four traits viz., days to first flower open, days to 50\%flowering, days to physiological maturity and height of first siliqua for cluster III was noticed which comprises of 11 genotypes (Table 1). But in terms of lowest mean values i. e. for earliness for days to first flower open, days to 50\%flowering and days to physiological maturity and lower placement of first primary branch and first siliqua along with lower angle of branch and also lower angle of siliqua for cluster VIII unique genotype Rajendra Suphlam exhibited its worth. In Euclidean method (Table 5) on basis of highest cluster mean harvest index and dry matter efficiency falls in cluster VII (PusaMahak) and for rest of important component traits i. e. primary branches plant ${ }^{1}$, secondary branches plant $^{-1}$, number of siliqua plant ${ }^{-1}$, length of siliqua, stem girth, internode length, height of the plant, number of siliqua in primary mother axis, angle of branch, angle of siliqua, number of seeds siliqua $^{-1}$, root volume, root length, root girth, 1000 seed weight, biological yield, oil content and grain yield /plant it falls in cluster VIII (Rajendra Suphlam) the oligo-genotypic cluster (Fig. 3 and 4).

On comparison between both methods based on cluster mean values and on per se performance lowest mean values for days to first flower open, days to $50 \%$ flowering, days to physiological maturity, angle of branch and angle of siliqua Pusa Mahak was superior genotype; whereas maximum mean values for 
length of siliqua, stem girth, internode length, height of the plant, number of siliqua in primary mother axis, height of first primary branch, height of first siliqua, number of seeds siliqua ${ }^{-1}$, root volume, root length, root girth, 1000 seed weight, biological yield, oil content and grain yield/plant cluster VIII genotype Rajendra suphlam was superior. This suggests that overall, on cluster mean basis, Rajendra suphlam is an early maturing genotypes shows compact and semi appressed angle of branch and siliqua respectively and also characters like deep and voluminous root system which makes suitable for drought condition. Rajendra suphlam also shows maximum cluster mean values for most of the characters like stem girth, internode length, height of the plant, number of seeds siliqua $^{-1}, 1000$ seed weight, biological yield, oil content and grain yield /plant which suggested usefulness of material for hybridization. Similar results were observed by Patel and Patel (2006), Singh et al., (2007), Zaman et al., (2010), Mahmud et al., (2012), Binod Kumar and Anil Pandey (2012).

Root length (45. 47\%) followed by height of first primary branch (25.71) and root volume (14. 29) characters covered 85. $39 \%$ contribution (Fig. 5) and were found maximum contributing characters towards total divergence. Similar observation by Zaman et al., (2010) and Doddabhimappa et al., (2010). This suggests, under rainfed condition, genotypes with superiority in root traits like deep tap rooted with more volume can provide more capacity to absorb water under moisture stress conditions in rainfed situation. Rajendra suphlam proved its worth as the genotype best suited with deep tap rooted along with more root volume, having spreaded capillary system advantage and least height of primary branch initiation providing more productive area from bottom to top for rainfed agro- ecologies of Bihar.

\section{Acknowledgement}

Authors are thankful to different All India Coordinated Research Project-Rapeseed and Mustard centres namely, DRMR, Bharatpur, Rajasthan, CCSHAU, Hisar, Haryana, BARC, Trombay, Maharastra, GBPUAT, Pantnagar, Uttarkhand, CSAUAT, Kanpur, U. P, IARI, New Delhi, ARS, RAU, Sriganganagar, Rajasthan, NDUAT, Faizabad, U. P and BAU, Kanke, Ranchi, Jharkhand, for providing genotypes of rapeseed and mustard.

\section{References}

Anand, I. J. and D. S. Rawat. 1984. Genetic diversity, combining ability and heterosis in brown mustard. Indian $J$. Genet. Pl. Breed. 44 (2): 226-234.

Ashana, A. N. and V. K. Pandy. 1980. Genetic divergence in linseed. Indian J. Genet. Pl. Breed. 40: 247-250.

Beale EML (1969). Euclidean cluster analysis. A paper contributed to 37th session of the Indian National Statistical Institute.

Doddabhimappa, R., Gangapur, B., Prakash G. and Channayya. P. H. 2010. Genetic Diversity Analysis of Indian Mustard (Brassica juncea L.) Electronic J. Pl. Breed. ; 1 (4): 407-413.

Katyal JC, Doshi SP, Malhotra PK (1985). Use of cluster analysis for classification of Benchmark soil samples from India in different micronutrient availability group. J. Agric. Sci. Cambridge 104:421-424

Khachatourians GG, Summer AK, Philips PWB 2001 An introduction to the history of canola and the scientific basis for innovation. CABI, London.

Khan, M. N. 2000. Multivariate analysis in raya (Brassica juncea). Applied Biological Research, 2 (1): 169-171.

Kumar, Binod and Pandey, A. 2013. Diversity Analysis in Indian mustard (Brassica 
juncea L. Czern and Coss). Madras Agric. J., 100 (1-3): 62-66.

Kumar, Rajesh; Haider, Z. A. and Sahai, V. N. 2000b. Genetic divergence in mutant lines of Indian mustard (Brassica juncea) cv varuna. Journal of Applied Biology, 10 (2): 142-144.

Kumar, Rajesh; Haider, Z. A.; and Sahai, V. N. 2000a. Genetic diversity in mutant lines of Indian mustard (Brassica juncea). Journal of Applied Biology, 10 (1): $1-4$

Mahalanobis, P. C., 1936. On the generalized distance in statistics. Proc. Natl. Acad. Sa., Indian, 12: 49-55.

Mahmud, F., Bhuiyan, S. R. and Rahim, A. 2012. Genetic Divergence in Advanced Lines of Oilseed Rape (Brassica napus ssp oleifera L.) Agriculturae Conspectus Scientificus; 77 (2) 81-85.

Patel, J. M. and Patel, K. M. 2006. Genetic divergence in Indian mustard (Brassica juncea L.). Indian J. Genet. Pl. Breed. ; 66 (1): 49-50.

Rao, C. R., 1952, Advanced Statistical Methods in Biometrieal Research. John Wiley and Sons, Inc., New York, pp. 357-363.

Shalini, T. S. 1998. Genetic divergence in Indian mustard [Brassica juncea L. (Czern and Coss)]. M. Sc. Thesis, University of Agricultural Science, Bangalore
Singh, H. 1986. Genetic variability, heritability, and drought indices analysis in Brassica species. J. Oilseeds Res. 3 (2):170-177.

Singh, R. K. and Chaudhary, B. D., 1977, Biometrical Methods in Quantitative Genetic Analysis. Kalyani publishers, New Delhi, p. 266.

Singh, V., Bhajan R. and Kumar, K. 2007. Genetic diversity in Indian mustard (Brassica juncea L. Czern and Coss.). Prog. Agric.; 7 (1/2): 105-109.

Tomooka, N. 1991. Genetic diversity and landrace differentiation of mungbean (Vigna radiata). An evaluation of its wild relatives as breeding materials. Tech. Bull. Trop. centre, Japan No. 28. Ministry of Agroforestry and Fisheries, Japan.

Uddin, M. J. and M. A. Z. Chowdhury. 1994. Genetic divergence in mustard. Bangladesh J. Genet. Pl. Breed. 7(2): 23-27.

Verma, S. K. and Sachan, J. N. 2000. Genetic divergence in Indian mustard (Brassica juncea (L.) Czern and Coss.). Crop Research, 19(2): 271-276.

Zaman, M. A., Khatun, M. T., Ullah, M. Z., Moniruzzamn, M. and Rahman, M. Z. 2010. Multivariate analysis of Divergence in advanced lines of mustard (Brassica spp). Bangladesh J. Pl. Breed. Genet., 23(2): 29-34

\section{How to cite this article:}

Khushboo Chandra, Anil Pandey and Mishra, S.B. 2018. Genetic Diversity Analysis among Indian Mustard (Brassica juncea L. Czern \& Coss) Genotypes under Rainfed Condition. Int.J.Curr.Microbiol.App.Sci. 7(03): 256-268. doi: https://doi.org/10.20546/ijcmas.2018.703.030 